\title{
Endometriosis in Adolescents
}

\author{
Erica C. Dun, MD, MPH, Kimberly A. Kho, MD, MPH, Vadim V. Morozov, MD, \\ Susan Kearney, MHSE, Jonathan L. Zurawin, MD, Ceana H. Nezhat, MD
}

\begin{abstract}
Background and Objectives: Women with endometriosis often report onset of symptoms during adolescence; however, the diagnosis of endometriosis is often delayed. The aim of this study was to describe the experience of adolescents who underwent laparoscopy for pelvic pain and were diagnosed with endometriosis: specifically, the symptoms, time from onset of symptoms to correct diagnosis, number and type of medical professionals seen, diagnosis, treatment, and postoperative outcomes.
\end{abstract}

Methods: We reviewed a series of 25 females $\leq 21$ years of age with endometriosis diagnosed during laparoscopy for pelvic pain over an 8-year period. These patients were followed up for 1 year after surgery.

Results: The mean age at the time of surgery was 17.2 (2.4) years (range, 10-21). The most common complaints were dysmenorrhea (64\%), menorrhagia (44\%), abnor$\mathrm{mal} /$ irregular uterine bleeding $(60 \%), \geq 1$ gastrointestinal symptoms (56\%), and $\geq 1$ genitourinary symptoms ( $52 \%$ ). The mean time from the onset of symptoms until diagnosis was 22.8 (31.0) months (range, 1-132). The median number of physicians who evaluated their pain was 3

Department of Obstetrics, Gynecology, and Reproductive Science, Yale School of Medicine, New Haven, Connecticut, USA (Dr. Dun).

Department of Obstetrics and Gynecology, University of Texas Southwestern Medical Center, Dallas, Texas, USA (Dr. Kho)

Department of Obstetrics, Gynecology, and Reproductive Sciences, University of Maryland School of Medicine, Baltimore, Maryland, USA (Dr. Morozov).

Atlanta Center for Minimally Invasive Surgery and Reproductive Medicine, Atlanta, Georgia, USA (Drs. Kearney, Nezhat).

Baylor College of Medicine, Houston, Texas, USA (Mr. Zurawin).

Stanford University College of Medicine, Stanford, California, USA (Dr. Nezhat).

Disclosures: Dr. Nezhat is a medical advisor to Plasma Surgical, Roswell, GA, and a consultant to Karl Storz Endoscopy America, Inc., El Segundo, CA, and is on the Scientific Advisory Board of SurgiQuest, Milford, CT.

Address correspondence to: Ceana H. Nezhat, MD, Atlanta Center for Minimally Invasive Surgery and Reproductive Medicine, 5555 Peachtree Dunwoody Road, Suite 276, Atlanta, GA 30342, USA. Telephone: 404-255-8778, Fax: 404- 255-5627, E-mail: ceana@nezhat.com

DOI: $10.4293 /$ JSLS.2015.00019

(C) 2015 by JSLS, Journal of the Society of Laparoendoscopic Surgeons. Published by the Society of Laparoendoscopic Surgeons, Inc.
(2.3) (range, 1-12). The adolescents had stage I (68\%), stage II (20\%), and stage III (12\%) disease. Atypical endometriosis lesions were most commonly observed during laparoscopy. At 1 year, 64\% reported resolved pain, 16\% improved pain, $12 \%$ continued pain, and $8 \%$ recurrent pain.

Conclusions: Timely referral to a gynecologist experienced with laparoscopic diagnosis and treatment of endometriosis is critical to expedite care for adolescents with pelvic pain. Once the disease is diagnosed and treated, these patients have favorable outcomes with hormonal and nonhormonal therapy.

Key Words: Adolescent endometriosis, Atypical endometriosis, Endometriosis, Pelvic pain, Referral pattern.

\section{INTRODUCTION}

Endometriosis, defined as endometrial tissue implanted outside the uterus, has been estimated to affect $10 \%$ to $15 \%$ of all reproductive-age women ${ }^{1}$ and $70 \%$ of women with chronic pelvic pain. ${ }^{2}$ Less established are the rates of laparoscopically confirmed endometriosis among adolescent females with pelvic pain; these estimates range from $19 \%$ to $73 \% .^{3-5}$ One prospective study, estimated the prevalence of endometriosis among adolescents with pelvic pain who underwent laparoscopy at $47 \%,{ }^{6}$ and 2 retrospective studies found endometriosis in $70 \%$ to $73 \%$ of adolescents with pelvic pain that was unresponsive to medical therapy. ${ }^{7,8}$

Although most women with endometriosis report the onset of symptoms during adolescence, diagnosis is often delayed. 9 Consequently, this delay may decrease their reproductive potential and functional outcomes. Moreover, early identification and treatment of endometriosis may resolve pain, prevent disease progression and organ damage, and preserve fertility. ${ }^{10}$

Adolescents with pelvic pain may present a diagnostic challenge, because they describe acyclic pain as well as cyclic pain and may present with an array of confounding symptoms. 9 The intraoperative appearance of en- 
dometriosis in adolescents may differ from the classic "powder-burn" lesions typically seen in adults. ${ }^{11}$ Furthermore, the health care costs associated with adolescent endometriosis are significant, not to mention the social and emotional costs to the girls caused by absenteeism at school and inability to participate in normal activities. ${ }^{12}$

In this study, we describe the experience of adolescent females with severe pelvic pain that was refractory to medical management, who underwent laparoscopy, during which endometriosis was ultimately diagnosed. We characterize the constellation of symptoms that led to incorrect diagnoses, time from onset of symptoms to diagnosis, type, and number of different medical professionals seen before diagnosis, prevalence of endometriosis in the population, surgical findings, and postoperative outcomes at 1 year.

\section{MATERIALS AND METHODS}

\section{Study Design}

This retrospective case series included consecutive adolescent females with pelvic pain who underwent laparoscopy and received a visual and histologic diagnosis of endometriosis at our tertiary surgical referral center between January 1, 2001, and December 31, 2009. Inclusion criteria were age 21 years or younger, pelvic pain refractory to medical management, and a history of multipuncture laparoscopy for pelvic pain. Exclusion criteria were previous surgical diagnosis of endometriosis and preoperative radiographic evidence of endometriosis (i.e., endometriomas). Those 2 populations were excluded because we wanted to establish the prevalence of endometriosis in adolescents without historical or radiographic evidence of disease. Figure 1 describes the selection process for the study.

Preoperative demographic and clinical data were obtained from the patients' medical records. Demographic data included age, body mass index (BMI), and race. Clinical data included gravidity, parity, age at menarche, coital status, family history of endometriosis (defined as a first- or second-degree relative with diagnosed endometriosis), and preoperative patient symptoms. Information regarding referral sources to our tertiary care center, time from the onset of symptoms until diagnosis of endometriosis, time from menarche until diagnosis, time from the first physician visit until diagnosis, number and specialties of physicians seen, prior diagnoses, prior trials of medical

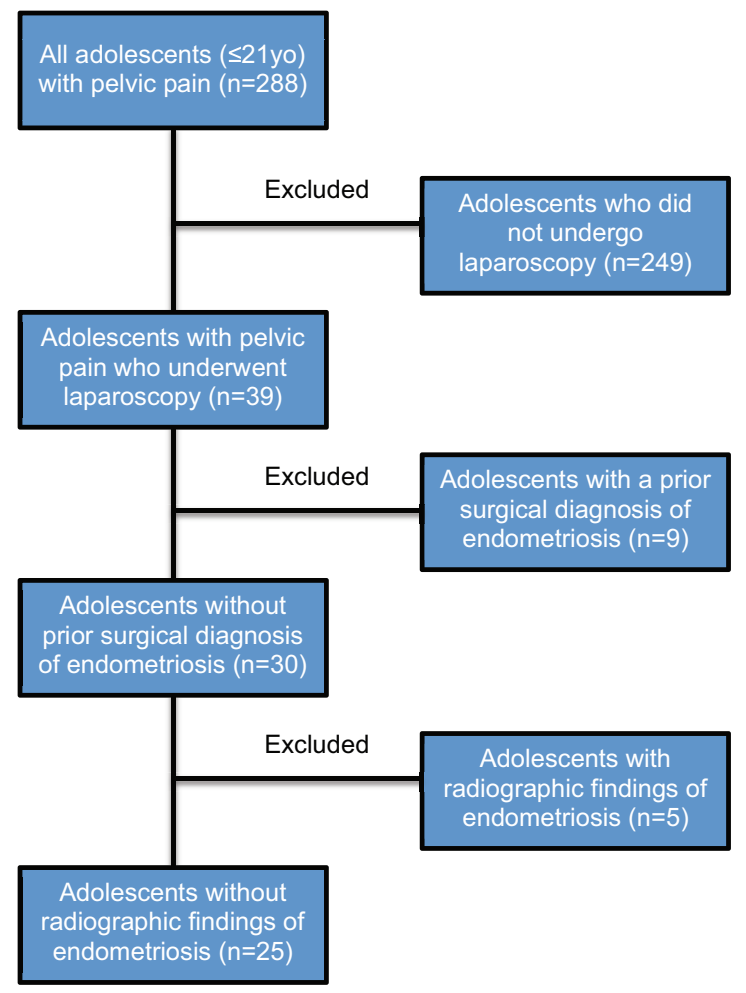

Figure 1. The selection process for the study.

therapy, and prior surgery were also obtained from the patients' medical records. The detailed information about severity and duration of symptoms, and previous diagnoses and treatments were obtained in a standard history obtained during the first office visit.

All patients who underwent laparoscopy had a preoperative diagnosis of severe pelvic pain that was refractory to medical treatment. They underwent multipuncture laparoscopy as previously described.13 One surgeon (C.H.N.) performed all the procedures. Endometriosis was diagnosed by visual inspection and by histopathologic analysis of biopsy specimens. The surgical treatment consisted of a combination of excision and ablative techniques to achieve maximal removal of the lesions. The severity of disease was staged according to the revised American Fertility Society classification system. ${ }^{14}$ Postoperative clinical information regarding postoperative medical treatment if any, subsequent abdominal and pelvic pain, subsequent surgeries for endometriosis or other etiologies, attempted pregnancies, and pregnancies were retrospectively obtained from the patients' medical records. Postoperative follow-up was assessed at 1 year. This study was ruled exempt by the Institutional Review Board at Northside Hospital (Atlanta, Georgia). 


\section{Statistical Analysis}

Descriptive statistics were used to analyze the data. Results were reported as the mean, (SD) and range of values, or number (n) and percent (\%) of the study group. Analyses were performed with the statistical software package SPSS 22.0 (IBM, Armonk, New York).

\section{RESULTS}

Two hundred eighty-eight adolescent females with pelvic pain were initially identified. Two hundred forty-nine were excluded because laparoscopy was not performed, 39 underwent laparoscopy for pelvic pain; 9 were excluded because they had prior surgical diagnosis of endometriosis, and 5 were excluded because of radiographic evidence of disease (i.e., endometriomas). Twenty-five patients met the inclusion and exclusion criteria and were analyzed in this case series. Figure 1 traces the selection process. The mean age at the time of surgery was 17.2 (2.4) years (range, 10-21). Fifty-six percent had a family history of endometriosis. Other demographic and preoperative clinical characteristics of the patients are described in Table 1. The most common preoperative complaints (Table 2) were dysmenorrhea (64\%), menorrhagia (44\%), abnormal or irregular uterine bleeding (60\%), at least 1 gastrointestinal symptom (56\%), and at least 1 genitourinary symptom $(52 \%)$.

Table 3 describes the referral patterns and prior diagnoses. On average, the amount of time between the first physician visit for pelvic pain and diagnosis was 10.9 months (22.0) (range, 1-108). The median number of physicians from different specialties who evaluated the patients' pelvic pain was 3 (2.3) (range, 1-12), and included $\geq 1$ obstetrician/gynecologists $(72 \%)$, gastroenterologists (36\%), urologists (16\%), and other specialists, including orthopedic surgeons, infectious disease physicians, pain management specialists, physical therapists, and psychiatrists. Eleven of the 25 (44\%) adolescents had been to the emergency department at least 1 time because of pelvic pain. The patients had diagnoses of other illnesses, including pelvic inflammatory disease (PID) (20\%), irritable bowel syndrome (IBS) and gastritis (16\%), ovarian cysts (12\%), musculoskeletal pain (12\%), and appendicitis (12\%). The patients' mothers (44\%) provided the primary referral to our tertiary care center, followed by another obstetrician/gynecologist (24\%), and friends of the patients' family (16\%).

In all 25 patients, endometriosis was diagnosed during the laparoscopy. Eighteen (72\%) had biopsy-proven endome-
Table 1.

Patient Demographics and Clinical Information

\begin{tabular}{ll}
\hline Variable & Data \\
\hline Patients, $n$ & 25 \\
Age at surgery, years & $17.2(2.4) ; 10-21$ \\
BMI, $\mathrm{kg} / \mathrm{m}^{2}$ & $23.0(3.9) ; 19-35$ \\
Race, $\mathrm{n}(\%)$ & $23(92 \%)$ \\
$\quad$ White & $2(8 \%)$ \\
$\quad$ Black & $0(0.4) ; 0-2$ \\
Gravidity & $0(0.4) ; 0-2$ \\
$\begin{array}{l}\text { Parity } \\
\text { Sexually active, } \mathrm{n}(\%)\end{array}$ \\
$\quad$ Yes & $8(32 \%)$ \\
$\quad$ No & $17(68 \%)$ \\
Family history of endometriosis, (\%) & \\
$\quad$ Yes & $14(56 \%)$ \\
$\quad$ No & $11(44 \%)$ \\
Age at menarche, years & $12.2(1.1) ; 10-14$ \\
Time from menarche until diagnosis, \\
months
\end{tabular}

BMI, body mass index. Unless otherwise noted, data are presented as the mean (SD); range.

triosis, and 7 (28\%) had visual diagnosis of endometriosis, but extensive biopsies were not performed because of the anatomic location of the endometriosis. Most of the patients in whom diagnosis was visual had fibrotic clear vesicular lesions and peritoneal defects. Most of the adolescents had stage I (68\%) endometriosis, follow by stage II (20\%), and III (12\%), according to the Revised American Society for Reproductive Medicine classification of endometriosis. ${ }^{14}$ None of the adolescents had stage IV endometriosis or evidence of extragenital endometriosis. The types of endometriosis lesions most commonly visualized were peritoneal defects (Figure 2) and atypical white/ fibrotic (Figure 3), clear (Figure 4), ovarian or cortical (Figure 5), hemosiderin/pigmented (Figure 6), and hemorrhagic (Figure 7). Table $\mathbf{4}$ describes the perioperative patient findings.

After the laparoscopy, most of the patients were given one or more medications, most commonly combined oral contraceptives (COCs) (64\%) and nonsteroidal antiinflammatory drugs (NSAIDs) (32\%). At 1 year, 64\% reported resolved pain, $16 \%$ reported improved pain, 
Table 2.

Preoperative Subjective Symptoms ${ }^{\mathrm{a}}$

\begin{tabular}{ll}
\hline Symptom & $\mathrm{n}(\%)$ \\
\hline Dysmenorrhea & $16(64)$ \\
Menorrhagia & $11(44)$ \\
Abnormal/irregular uterine bleeding & $15(60)$ \\
Dyspareunia & $4(16)$ \\
Genitourinary symptoms & \\
Bladder pain & $1(4)$ \\
Flank pain & $1(4)$ \\
Back pain & $6(24)$ \\
Dysuria & $4(16)$ \\
Urinary frequency and urgency & $9(36)$ \\
Urinary incontinence & $2(8)$ \\
Hematuria & $2(8)$ \\
Nocturia & $3(12)$ \\
Gastrointestinal symptoms & \\
Nausea & $11(44)$ \\
Constipation & $5(20)$ \\
Diarrhea & $6(24)$ \\
Dyschesia & $7(28)$ \\
Hematochesia & $3(12)$ \\
Constitutional symptoms & \\
Fatigue & $6(24)$ \\
Acne & $5(20)$ \\
Headache & $2(8)$ \\
Insomnia & $1(4)$ \\
Premenstrual dysphoric disorder & $6(24)$ \\
Depression & $1(4)$ \\
\hline a Patients may have had more than 1 symptom. \\
\hline \hline
\end{tabular}

$12 \%$ reported continued pain, and $8 \%$ stated that the pain had initially improved but then had returned. Table 5 summarizes the postoperative treatments and pain outcomes at 1 year.

\section{DISCUSSION}

In a survey of more than 4000 women reporting surgically diagnosed endometriosis, two-thirds of the respondents experienced symptoms during adolescence. Those women were far more likely to be told by their physicians that nothing was wrong, as opposed those who sought treatment for symptoms that started later in life. ${ }^{9}$
Table 3.

Referral Patterns and Diagnoses

\begin{tabular}{ll}
\hline Variable & Data \\
\hline Time from first physician visit until diagnosis, & $10.9(22.0) ;$ \\
months & $1-108$ \\
Median number of physicians seen before diagnosis & $3(2.3) ; 1-12$ \\
Type of physicians seen before diagnosis, $\mathrm{n}(\%)^{\mathrm{a}}$ & \\
Obstetrician/gynecologist & $18(72)$ \\
Emergency room physician & $11(44)$ \\
Gastroenterologist & $9(36)$ \\
Urologist & $4(16)$ \\
Internal medicine physician & $4(16)$ \\
General surgeon & $3(12)$ \\
Pediatrician & $3(12)$ \\
None & $3(12)$ \\
Other & $6(24)$
\end{tabular}

Prior diagnoses, $\mathrm{n}(\%)^{\mathrm{a}}$

Endometriosis, not surgically diagnosed 5 (20)

Pelvic inflammatory disease 5 (20)

Irritable bowel syndrome/gastritis 4 (16)

Dysmenorrhea 4 (16)

Appendicitis 3(12)

Ovarian cysts 3(12)

Musculoskeletal pain $3(12)$

Renal colic/interstitial cystitis 2 (8)

Crohn's disease 2 (8)

None 4 (16)

Other 3 (12)

Average number of months on COCs $(n=17) \quad 13.5(20.6)$;

$1-84$

Average number of months on NSAIDs $(n=10) \quad 7.5$ (9.1);

$1-24$

History of prior surgery $(\mathrm{n}=25), \mathrm{n}(\%)$

Yes $3(12)$

No 22 (88)

Referral source $(\mathrm{n}=25), \mathrm{n}(\%)$

Mother 11 (44)

Obstetrician/gynecologist $6(24)$

Friend of the patient's family 4 (16)

Internal medicine physician 2 (8)

Emergency room physician 1 (4)

Nutritionist 1 (4)

a Patients may have had more than 1 doctor or diagnosis. Unless otherwise noted, data are presented as the mean (SD); range. 


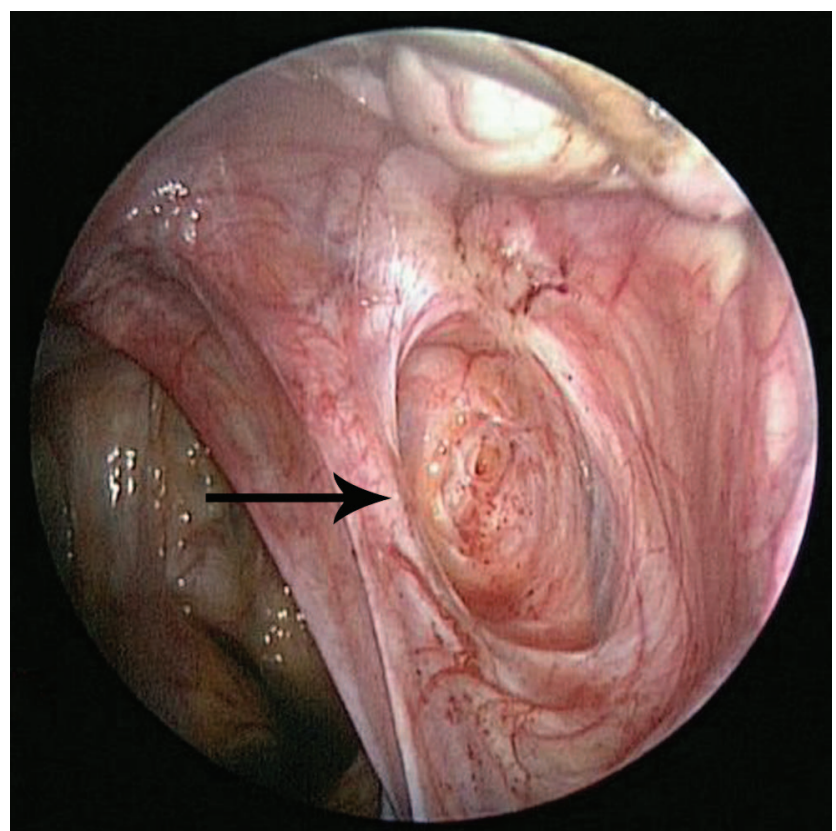

Figure 2. Peritoneal defect (arrow) with red, punctate lesions in the center of the defect in the right ovarian fossa in a 16-year-old patient with stage II endometriosis.

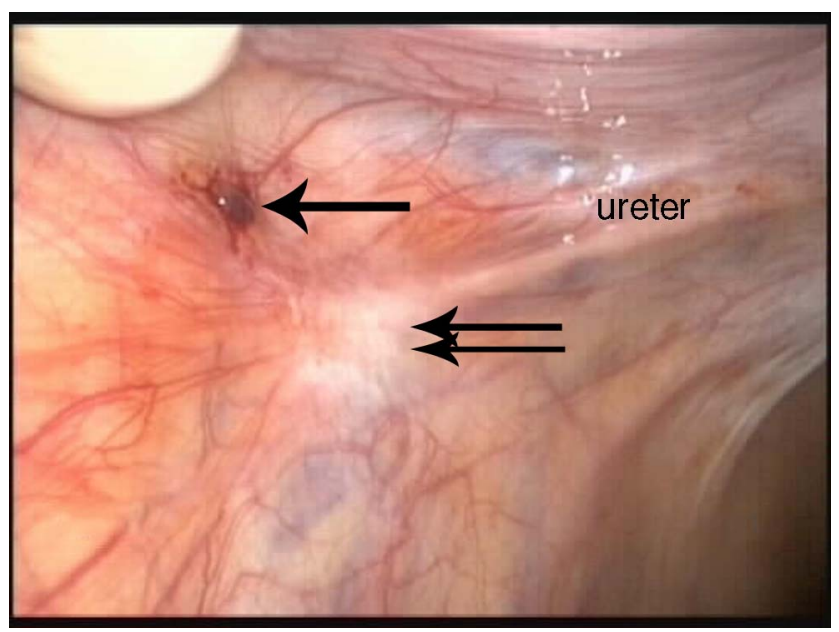

Figure 3. Hemorrhagic endometriosis (arrow) involving the left pelvic sidewall with an atypical white fibrotic endometriosis lesion (double arrow) immediately over the left ureter and left ovarian fossa in a 16-year-old patient with stage II endometriosis.

Our study suggests that adolescents are overlooked because they may present with atypical symptoms of endometriosis. Vague abdominal symptoms, gastrointestinal distress, and genitourinary symptoms can confound the diagnosis of endometriosis, especially when the first health care provider the patient sees is not a gynecologist. In our cohort, the adolescents had an average 23-month

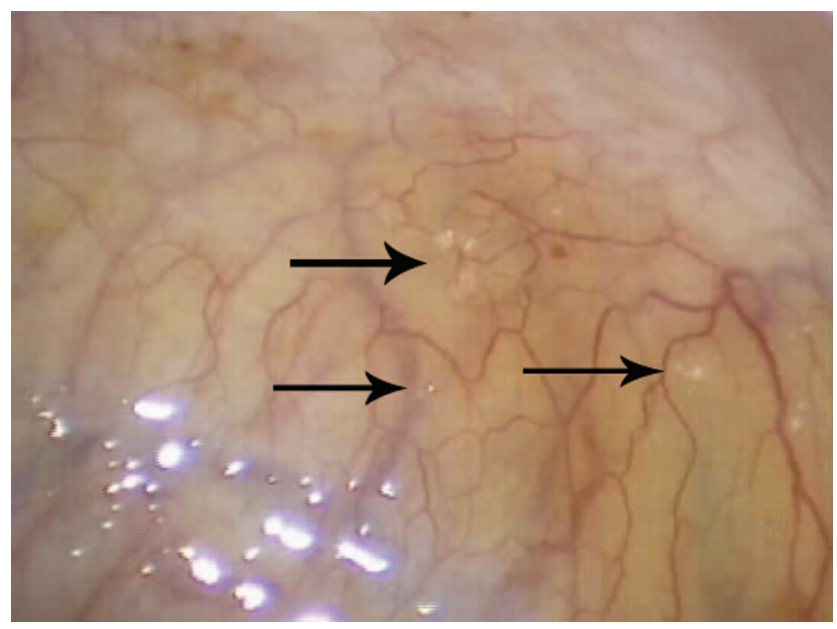

Figure 4. Atypical clear endometriosis lesions studding the peritoneum of the posterior cul-de-sac. The clear, vesicular, superficial lesions (arrow) were found in an 18-year-old patient with stage I endometriosis who had a family history of the disease.

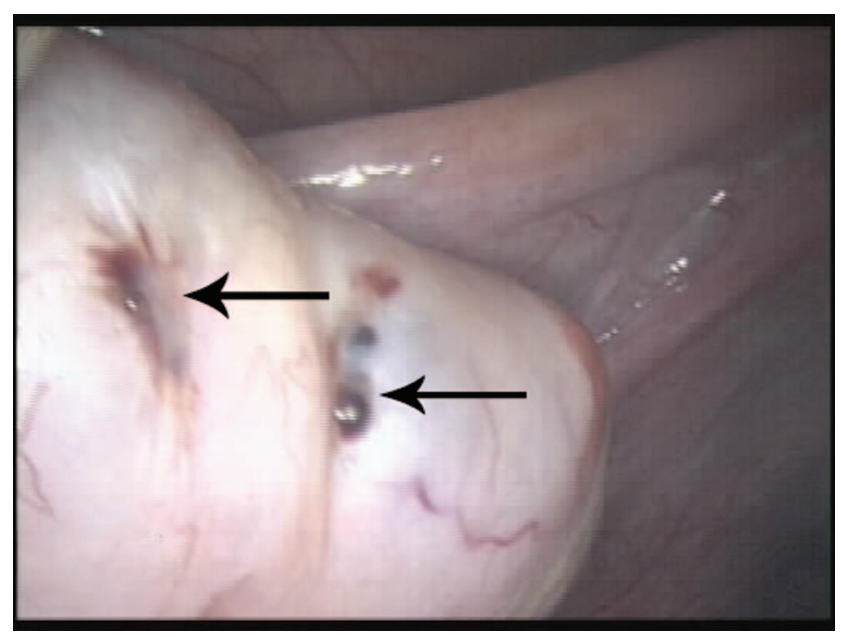

Figure 5. Cortical endometriosis lesions (arrow) on the left ovary in a 15 -year-old patient with stage III endometriosis.

delay in diagnosis from the onset of symptoms. Even after medical care was sought, almost a year was spent seeking appropriate diagnosis and treatment. Patients and their families appeared to self-advocate, with $44 \%$ of the referrals coming from the patients' mothers. This finding also highlights the potentially strong familial component of endometriosis, with $56 \%$ of patients reporting a positive family history in this patient sample. ${ }^{15}$

Beyond missed school days and activities, adolescent females can be misdiagnosed with such conditions as PID and IBS, which can have adverse psychological impacts on them and may color their experiences with the health 
Endometriosis in Adolescents: Referrals, Diagnosis, Treatment, and Outcomes, Dun EC et al.

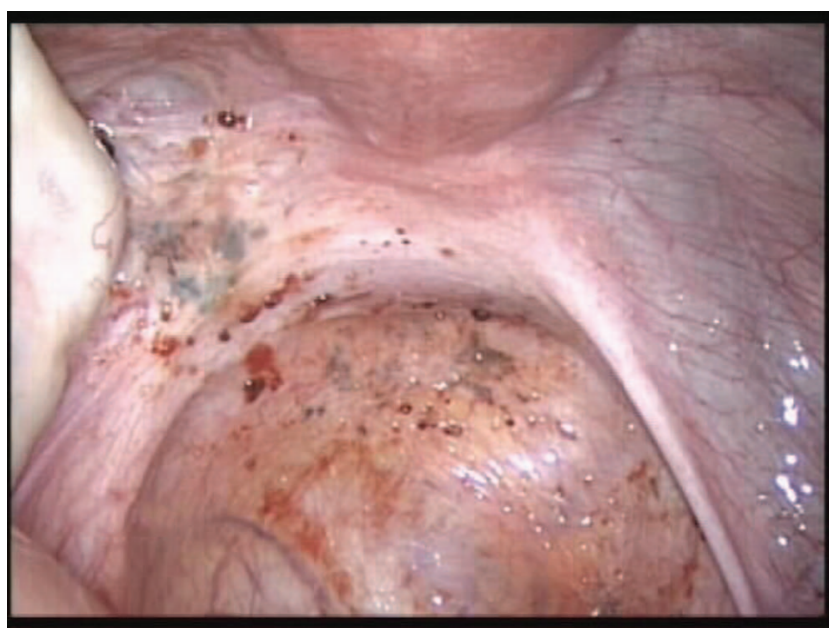

Figure 6. Scattered variable-appearing endometriosis lesions with severe disease in the left pelvis. The endometriosis invaded the retroperitoneal fibroadipose tissue of the left posterior cul-desac and also involved the left paraureteral region. This extensive disease presentation was found in a 15 -year-old patient with stage III endometriosis (the patient shown in Figure 5).

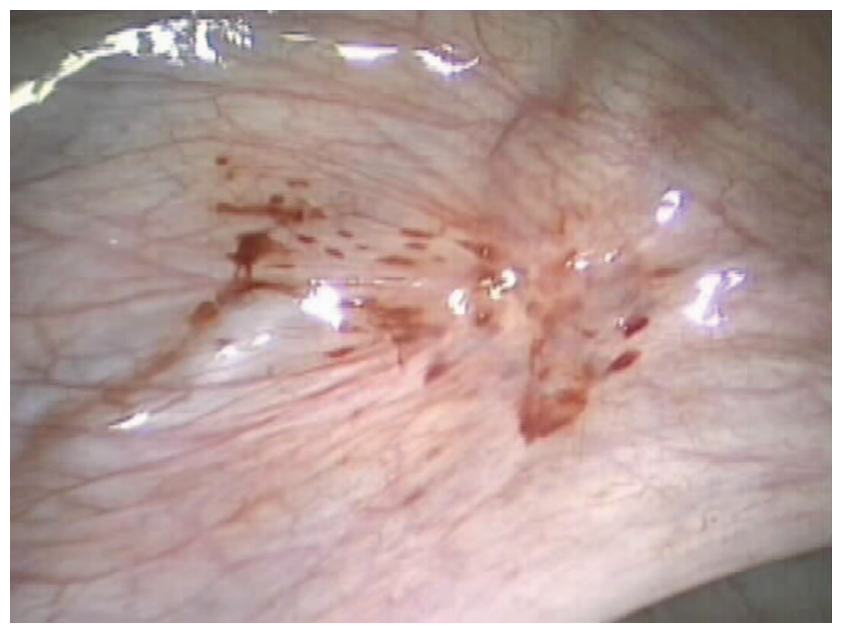

Figure 7. Hemorrhagic red endometriosis lesions involving the left broad ligament and perivesicular peritoneum in a 20 -yearold patient with stage II endometriosis.

care system and how future health care providers view them. In our case series, the adolescents were evaluated on average by 3 physicians and referred to specialists such as psychiatrists and orthopedic surgeons before endometriosis was diagnosed. The delay in diagnosis decreased when the first physician who evaluated them was an obstetrician/gynecologist.

We advocate a see-and-treat approach for young women in whom medical therapy for dysmenorrhea has failed,
Table 4.

Perioperative Outcomes

\begin{tabular}{ll}
\hline Variable & $\mathrm{n}(\%)$ \\
\hline Endometriosis stage $(\mathrm{n}=25)$ & $17(68)$ \\
$\quad$ I & $5(20)$ \\
II & $3(12)$ \\
III & 0 \\
IV & \\
Types of endometriosis lesions ${ }^{\mathrm{a}}$ & $13(68)$ \\
Peritoneal defects & $11(44)$ \\
Atypical white/fibrotic & $6(24)$ \\
Atypical clear & $5(20)$ \\
Ovarian/cortical lesions & $5(20)$ \\
Hemosiderin/pigmented & $4(16)$ \\
Hemorrhagic & $3(12)$ \\
Miliary/nodular & $2(8)$ \\
Vesicular/endosalpingiosis & \\
\hline${ }^{\text {Patients may have had more than } 1 .}$ \\
\hline \hline
\end{tabular}

Table 5.

Outcomes at 1 Year

\begin{tabular}{ll}
\hline Outcome & $\mathbf{n}(\mathbf{\%})$ \\
\hline Postoperative medical treatment ${ }^{\mathrm{a}}$ & \\
COCs & $16(64)$ \\
Progestins & $3(12)$ \\
NSAIDs & $8(32)$ \\
None & $3(12)$ \\
Pain symptoms & \\
$\quad$ Successfully resolved pain & $16(64)$ \\
Improved pain & $4(16)$ \\
Continued pain & $3(12)$ \\
Recurrent pain & $2(8)$ \\
Length of follow up (months) & $20.0(18.6) ; 0.5-58$
\end{tabular}

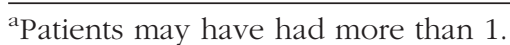

${ }^{\mathrm{b}}$ Data are presented as the mean (SD); range.

because the prevalence of endometriosis is high in this population. Goals of surgical intervention are simultaneous diagnosis and conservative treatment to reduce the bulk of disease while decreasing pain and maintaining reproductive capacity. In our study, most of the adolescents had earlystage endometriosis, confined to the pelvis. This finding 
concurs with other studies that demonstrated that adolescent females have early-stage disease, $, 3,16,17$ that endometriosis may be a progressive disease, and that early diagnosis and ablation or removal of the affected tissue may decrease the long-term detrimental effects of the disease, including chronic pain and infertility. ${ }^{18,19}$

However, it should be noted that in adolescent females, subtle atypical lesions such as those that are clear, white, or red are more common ${ }^{4,20,21}$ and may be missed during laparoscopy if surgeons are looking for the powder-burn lesions that are commonly seen in adults. In fact, Vercellini et al. ${ }^{4}$ visually diagnosed endometriosis in $40 \%$ of adolescent females with pelvic pain, but this percentage rose to $52 \%$ when atypical clear or red vesicular lesions were included. Familiarity with atypical lesions may help the physician to arrive at the correct diagnosis among adolescents. We have observed that adolescent females have clinical improvements in their endometriosis-associated pain symptoms, with good pain control at the 1-year follow-up after laparoscopic diagnosis and concurrent treatment with either resection or ablation and postoperative medical therapy. At 1 year, $80 \%$ of the patients in our study reported either resolved or improved pain after surgery. Although we do not have 5-year follow-up data, Tandoi et al. ${ }^{22}$ reported a $56 \%$ recurrence rate at 5 years, and $34 \%$ of their adolescent cohort underwent a second laparoscopy to treat recurrent symptoms. ${ }^{22}$

The strength of our study is the number of adolescent females from our tertiary referral center and the inclusion of data regarding referral and prior diagnoses and treatment. It examines possible etiologies for the delay in diagnoses and emphasizes the atypical symptoms and atypical endometriosis lesions found in adolescents. The primary weaknesses are the retrospective study design and lack of ability to control the data collection including standardized pre- and postoperative validated pain assessments. The population itself, consisting of primarily white females at a tertiary referral center, limits the generalizability of the study. Recall bias is another limitation to data collection regarding the patients' previous diagnoses, physicians, and prior treatments. Another weakness is the varied postoperative treatments. This variation prevents us from differentiating whether the surgery and/or postoperative treatment affected the patients' postoperative pain outcomes. In addition, the descriptive nature of the case series precludes the ability to draw significant conclusions about our findings.

Future work will focus on prospective studies examining the relationship of early diagnosis and treatment with pain and fertility outcomes. We also hope to examine the association between diagnosis at an early age and a familial form of endometriosis, since many in the study had a positive family history of endometriosis. Finally, we would like to conduct a prospective study to learn whether excision versus ablation is associated with better postoperative pain and fertility outcomes.

\section{CONCLUSION}

Adolescents with severe pelvic pain and no historic or radiographic evidence of pathology have a high rate of endometriosis. Thus, endometriosis should be strongly considered in adolescent females with pelvic pain refractory to medical management. Nevertheless, it must be emphasized that medical management is the first-line therapy and only after a sufficient trial of medical therapy should surgical management with laparoscopic evaluation and treatmentbe pursued. The findings of this descriptive study suggest a potentially long and tortuous road to appropriate diagnosis and treatment. It also highlights the initial challenges that young females with endometriosis encounter. Therefore, timely referral to a gynecologist who is experienced with the laparoscopic diagnosis of endometriosis in adolescents and conservative treatment may significantly benefit their future quality of life.

\section{References:}

1. Giudice LC, Kao LC. Endometriosis. Lancet. 2004;364:17891799 .

2. Carter JE. Combined hysteroscopic and laparoscopic findings in patients with chronic pelvic pain. J Am Assoc Gynecol Laparosc. 1994;2:43-47.

3. American College of Obstetricians and Gynecologists. Endometriosis in adolescents, ACOG Committee Opinion Number 310. Obstet Gynecol. 2005;105:921-927.

4. Vercellini P, Fedele L, Arcaini L, Bianchi S, Rognoni MT, Candiani GB. Laparoscopy in the diagnosis of chronic pelvic pain in adolescent women. J Reprod Med. 1989;34:827-830.

5. Kontoravdis A, Hassan E, Hassiakos D, Botsis D, Kontoravdis N, Creatsas G. Laparoscopic evaluation and management of chronic pelvic pain during adolescence. Clin Exp Obstet Gynecol. 1999;26:76-77.

6. Goldstein DP, De Cholnoky C, Emans SJ. Adolescent endometriosis. J Adolesc Health Care1980;1:37-41.

7. Laufer MR, Goitein L, Bush M, Cramer DW, Emans SJ. Prevalence of endometriosis in adolescent girls with chronic pelvic pain not responding to conventional therapy. J Pediatr Adolesc Gynecol. 1997;10:199-202. 
8. Reese KA, Reddy S, Rock JA. Endometriosis in an adolescent population: the Emory experience. J Pediatr Adolesc Gynecol. 1996;9:125-128.

9. Greene R, Stratton P, Cleary SD, Ballweg ML, Sinaii N. Diagnostic experience among 4,334 women reporting surgically diagnosed endometriosis. Fertil Steril 2009;91:32-39.

10. Laufer MR. Current approaches to optimizing the treatment of endometriosis in adolescents. Gynecol Obstet Invest. 2008; 66(Suppl 1):19-27.

11. Laufer MR, Sanfilippo J, Rose G. Adolescent endometriosis: diagnosis and treatment approaches. J Pediatr Adolesc Gynecol. 16(3 Suppl):S3-S11, 2003.

12. Gao X, Outley J, Botteman M, Spalding J, Simon JA, Pashos CL. Economic burden of endometriosis. Fertil Steril. 2006;86: 1561-1572.

13. King LP, Nezhat $\mathrm{CH}$, Nezhat F, et al. Laparoscopic access. In: Nezhat's Video-Assisted and Robotic-Assisted Laparoscopy and Hysteroscopy. 4th ed. New York, NY: Cambridge University Press; 2013:41-53.

14. Revised American Society for Reproductive Medicine classification of endometriosis: 1996. Fertil Steril. 1997;67:817-821.

15. Dun EC, Taylor RN, Wieser F. Advances in the genetics of endometriosis. Genome Med. 2010;14:75-80.
16. Brosens I, Gordts S, Benagiano G. Endometriosis in adolescents is a hidden, progressive and severe disease that deserves attention, not just compassion. Hum Reprod. 2013;28:20262031.

17. Reese KA, Reddy S, Rock JA. Endometriosis in an adolescent population: the Emory experience. J Pediatr Adolesc Gynecol. 1997;9:125-128.

18. Dovey S, Sanfilippo J. Endometriosis and the adolescent. Clin Obstet Gynecol. 2010;53:420-428.

19. Janssen EB, Rijkers AC, Hoppenbrouwers K, Meuleman C, D'Hooghe TM. Prevalence of endometriosis diagnosed by laparoscopy in adolescents with dysmenorrhea or chronic pelvic pain: a systematic review. Hum Reprod Update. 2013;19:570582.

20. Redwine DB. Age-related evolution in color appearance of endometriosis. Fertil Steril. 1987;48:1062-1063.

21. Davis GD, Thillet E, Lindemann J. Clinical characteristics of adolescent endometriosis. J Adolesc Health. 1993;14:362-368.

22. Tandoi I, Somigliana E, Riparini J, Ronzoni S, Vigano P, Candiani M. High rate of endometriosis recurrence in young women. J Pediatr Adolesc Gynecol. 2011;24:376-379. 